\title{
What can National Registry data tell us about occult breast cancer?
}

\author{
John Benjamin Wild ${ }^{1}$, Steven Thrush ${ }^{2}$, Raghavan Vidya ${ }^{1}$ \\ ${ }^{1}$ Department of Oncoplastic Breast Surgery, New Cross Hospital, Royal Wolverhampton NHS Trust, Wolverhampton, UK; ${ }^{2}$ Department of \\ Oncoplastic Breast Surgery, Worcester Royal Hospital, Worcester, UK \\ Correspondence to: Raghavan Vidya, MS, MD, FRCS. Department of Oncoplastic Breast Surgery, New Cross Hospital, Royal Wolverhampton NHS \\ Trust, Wolverhampton, UK. Email: Raghavan.Vidya@nhs.net. \\ Comment on: Ge LP, Liu XY, Xiao Y, et al. Clinicopathological characteristics and treatment outcomes of occult breast cancer: a SEER population- \\ based study. Cancer Manag Res 2018;10:4381-91.
}

Submitted Jan 29, 2019. Accepted for publication Feb 15, 2019.

doi: $10.21037 /$ cco.2019.02.03

View this article at: http://dx.doi.org/10.21037/cco.2019.02.03

Axillary metastasis from an undetectable carcinoma of the breast is known as occult breast cancer $(\mathrm{OBC})$ and accounts for less than $1 \%$ of all breast cancer (1-3). Management of OBC initially favoured axillary lymph node dissection (ALND) with mastectomy (4). However, there have also been series reporting the use of ALND with breast conserving surgery (BCS), whole breast radiotherapy (WBRT) and observation of the breast (5-7). Due to its rarity, data on OBC is often from small series collected over long periods of time. National registry provides the opportunity to accrue larger numbers, determining more accurate incidence, and where possible generating epidemiological and clinicopathological data. The Surveillance, Epidemiological and End Results (SEER) database is a project of the American National Cancer Institute, the function of which is to routinely collect various cancer data on patients from various American states, covering $28 \%$ of the population.

The new analysis presented by Ge et al. reports the clinicopathological data within the SEER database on OBC (8). The authors compared characteristics of 479 patients with OBC and 115,739 non-OBC patients. Findings from the analysis demonstrated increased rates of stage III disease, N3 disease, oestrogen receptor (ER) and Progesterone Receptor (PR) negativity and Her2 receptor positivity. Furthermore, Ge et al. analysed survival and found that both overall survival (OS) and breast cancer specific survival (BCSS) were similar to the non-OBC group. However, in the 1:1 matched case control analysis of $\mathrm{OBC}$ and non-OBC cases there was significantly better overall and BCSS in the OBC group. This is the second time data from the SEER database relating to OBC has been utilised, however the previous publication did not undertake the propensity matching in order to draw novel conclusions on survival (3). These novel findings clearly raise some interesting questions-if there is a higher rate of ER negativity and Her 2 positivity why would these patients have improved survival in the 1:1 matched analysis? Ge et al. considered that discordance between primary tumours and nodal metastases may account for this. However, in the OBC there is not a primary lesion to analyse and therefore the nodal and receptor status may not reflect that of the initial lesion.

As there has been limited evidence published on OBC there remains uncertainty on optimum treatment strategy. Halsted advocated mastectomy and ALND in the initial report of OBC (9). This remained the mainstay of treatment for many years, however there are reports of utilising BCS, performing an upper-outer quadrantectomy as this was felt to represent the most likely location to find an OBC (10). The Korean Breast Cancer Society data showed that ALND \& BCS \& WBRT had comparable survival to Mastectomy \& ALND (1). A third interventional option is to utilise WBRT in addition to ALND (6). A survey of American breast surgeons conducted in 2005 demonstrated that in order to treat OBC $43 \%$ opted for mastectomy, $37 \%$ whole breast radiation and $6 \%$ would not treat the breast (11). Ge and colleagues were able to perform survival analysis dependent upon treatment. BCSS and OS where found to be similar when patients were treated with BCS \& ALND and WBRT or with mastectomy \& ALND (8). The authors assert that this is evidence that BCS with WBRT may be superior to mastectomy in the treatment of OBC. However, the BCSS and OS was also similar when 
these treatments where compared with the ALND only cohort. This is contrary to other analyses, where ALND alone was associated with lower rates of survival (2). One treatment modality associated with significantly worse survival was sentinel lymph node dissection (SLND), with OS at 100 months less than $50 \%$. Treatment modality was also evaluated in meta-analysis from Macedo et al. including 7 none randomized case control studies of around 200 patients with OBC (12). Findings of this meta-analysis include no significant difference between ALND \& WBRT and ALND \& mastectomy in terms of mortality, distant metastasis and locoregional recurrence. Ge et al. cannot provide data on locoregional recurrence as it is not recorded in the SEER database. However, Macedo et al. found significantly worse mortality and locoregional recurrence when ALND \& WBRT was compared to ALND alone, again contrary to the findings of Ge.

This study is another piece of evidence to state that OBC behaved differently to palpable or radiologically detected breast cancer, OBC patients have improved survival when compared to a matched cohort of non-OBC patient's. A common theme with all publications relating to $\mathrm{OBC}$ is the long period of data accrual (2004-2014) and the inevitable changes in clinical practice that will have occurred, in terms of, radiological techniques and technology, histopathological and molecular analysis, oestrogen modulating medications, chemotherapeutic regimes and biologically active monoclonal antibodies. The authors acknowledge the study limitations and emphasise the need for a large population based multi institutional analysis to enhance our understanding and improve the management of this rare presentation of breast cancer. Therefore, national registry data has allowed $\mathrm{Ge}$ et al. a large and relatively contemporaneous group of OBC patients to be analysed and determine that this rare form of breast cancer does not have the same clinicopathological features of non-OBC, OBC has improved survival when compared to matched non-OBC cases and furthermore the management of $\mathrm{OBC}$ may not require mastectomy, which many surgeons have considered the gold standard of treatment.

\section{Acknowledgments}

None.

\section{Footnote}

Conflicts of Interest: The authors have no conflicts of interest to declare.

\section{References}

1. Sohn G, Son BH, Lee SJ, et al. Treatment and survival of patients with occult breast cancer with axillary lymph node metastasis: a nationwide retrospective study. J Surg Oncol 2014;110:270-4.

2. Wang X, Zhao Y, Cao X. Clinical benefits of mastectomy on treatment of occult breast carcinoma presenting axillary metastases. Breast J 2010;16:32-7.

3. Walker GV, Smith GL, Perkins GH, et al. Populationbased analysis of occult primary breast cancer with axillary lymph node metastasis. Cancer 2010;116:4000-6.

4. Vezzoni P, Balestrazzi A, Bignami P, et al. Axillary lymph node metastases from occult carcinoma of the breast. Tumori 1979;65:87-91.

5. Vlastos G, Jean ME, Mirza AN, et al. Feasibility of breast preservation in the treatment of occult primary carcinoma presenting with axillary metastases. Ann Surg Oncol 2001;8:425-31.

6. Ellerbroek N, Holmes F, Singletary E, et al. Treatment of Patients With Isolated Axillary Nodal Metastases From an Occult Primary Carcinoma Consistent With Breast Origin. Cancer 1990; 66:1461-7.

7. Shannon C, Walsh G, Sapunar F, et al. Occult primary breast carcinoma presenting as axillary lymphadenopathy Breast 2002;11:414-8.

8. Ge LP, Liu XY, Xiao Y, et al. Clinicopathological characteristics and treatment outcomes of occult breast cancer: a SEER population-based study. Cancer Manag Res 2018;10:4381-91.

9. Halsted WS. I. The Results of Radical Operations for the Cure of Carcinoma of the Breast. Ann Surg 1907;46:1-19.

10. Svastics E, Rónay P, Bodó M. Occult breast cancer presenting with axillary metastasis. Eur J Surg Oncol 1993;19:575-80.

11. Khandelwal AK, Garguilo GA. Therapeutic options for occult breast cancer: a survey of the American Society of Breast Surgeons and review of the literature. Am J Surg 2005;190: 609-13.

12. Macedo FI, Eid JJ, Flynn J, et al. Optimal Surgical Management for Occult Breast Carcinoma: A Metaanalysis. Ann Surg Oncol 2016;23:1838-44.

Cite this article as: Wild JB, Thrush S, Vidya R. What can National Registry data tell us about occult breast cancer? Chin Clin Oncol 2019;8(Suppl 1):S15. doi: 10.21037/cco.2019.02.03 